\title{
The efficacy and safety of high-intensity focused ultrasound in the treatment of benign thyroid nodules: A systematic review and meta-analysis from 1990 to 2021
}

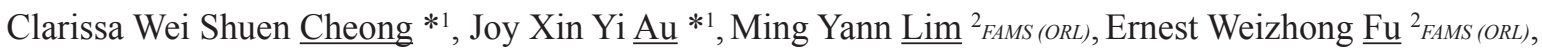

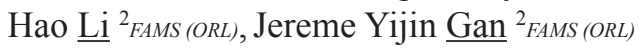

\begin{abstract}
Introduction: To date, there have only been 2 systematic reviews, and 1 systematic review and meta-analysis on high-intensity focused ultrasound (HIFU) for benign thyroid nodules. The present systematic review and meta-analysis seeks to evaluate the efficacy and safety of HIFU in the treatment of benign thyroid nodules.

Methods: PubMed, Embase and Cochrane databases were searched for relevant studies from 1990 to 2021. Nine studies were included in the systematic review and 6 in the meta-analysis. Pooled volume reduction rates (VRRs) at 3, 6 and 24 months after HIFU were assessed.

Results: This systematic review and meta-analysis showed that pooled VRRs at 3, 6, and 24 months after HIFU were 42.14 (95\% confidence interval [CI] 28.66-55.62, $\mathrm{I}^{2}=91 \%$ ), 53.51 (95\% CI 36.78-70.25, $\left.\mathrm{I}^{2}=97 \%\right)$ and $46.89\left(95 \%\right.$ CI $\left.18.87-74.92, \mathrm{I}^{2}=99 \%\right)$, respectively. There was significant heterogeneity in the pooled VRRs at 3, 6 and 24 months after HIFU. No studies recorded complete disappearance of the nodules. Common side effects included pain, skin changes and oedema. There were no major complications except for transient vocal cord paralysis and voice hoarseness $(0.014 \%)$ and transient Horner syndrome $(0.5 \%)$.

Conclusion: HIFU may be an effective and safe alternative treatment modality for benign thyroid nodules. Larger clinical trials with longer follow-up are needed to evaluate the effectiveness of HIFU in treating benign thyroid nodules.
\end{abstract}

Ann Acad Med Singap 2022;51:101-8

Keywords: Benign thyroid nodule, HIFU, high-intensity focused ultrasound, meta-analysis, minimally invasive technique, MITT, systematic review

\section{INTRODUCTION}

Thyroid nodules are commonly seen in clinical practice. They can be detected by palpation in $5 \%{ }^{1}$ of individuals and by ultrasound (US) in 19-68\% of the general population. ${ }^{2}$ Most of them are benign, stable and asymptomatic, thus preferred treatments are usually noninvasive. These would include modalities ranging from percutaneous ethanol injection (PEI), radiofrequency ablation (RFA) to high-intensity focused ultrasound (HIFU). ${ }^{3,4}$ The nature of the thyroid nodule determines the type of treatment indicated. While PEI is indicated for recurrent benign thyroid cysts, RFA is indicated for solid or cystic nodules or solid components of cystic nodules refractory to PEI. HIFU is indicated for solid or predominantly solid nodules ( $<30 \%$ cystic component). ${ }^{5}$

HIFU is an emerging non-invasive, US-guided thermal ablation technique that is being increasingly applied to the treatment of benign thyroid nodules. It has classically been used in other organs such as prostate, breast and liver. ${ }^{5}$ HIFU works through continuous low frequency ultrasonic waves and acoustic lenses are used to achieve the required intensity for target tissues

\footnotetext{
${ }^{1}$ Yong Loo Lin School of Medicine, National University of Singapore, Singapore

${ }^{2}$ Department of Otorhinolaryngology, Tan Tock Seng Hospital, Singapore

Correspondence: Dr Jereme Yijin Gan, Department of Otorhinolaryngology, Head and Neck Surgery, Tan Tock Seng Hospital, 11 Jalan Tan Tock Seng, Singapore 308433.

Email: Jereme_YJ_Gan@ttsh.com.sg

*Joint first authors
} 


\section{CLINICAL IMPACT}

\section{What is New}

- This review analysed the efficacy and safety of high-intensity focused ultrasound (HIFU) across a longer duration of follow-up, with subgroup and sensitivity analyses not found in previous studies.

- There was significant heterogeneity in the pooled volume reduction rates at 3, 6 and 24 months after HIFU. Common side effects included pain and skin changes.

\section{Clinical Implications}

- HIFU may be an effective, safe alternative treatment modality. Further studies with larger populations are required to assess the efficacy of HIFU based on size, type and location of nodule.

without damaging surrounding tissues. Its major advantage over other thermal techniques is that it could induce a focused thermal tissue destruction of up to $85^{\circ} \mathrm{C}$ without needing needle puncture and skin penetration. ${ }^{6}$ Side effects include pain that is usually transient and mild, ${ }^{5,7,8}$ skin blisters $^{5}$ and hoarseness. ${ }^{9}$

There have been only 2 systematic reviews ${ }^{3,5}$ and 1 systematic review and meta-analysis ${ }^{10}$ on HIFU and benign thyroid nodules to date. The present systematic review and meta-analysis seeks to evaluate the efficacy and safety of HIFU treatment for benign thyroid nodules, with the aim of updating the information presented in previous studies given the emergence of new studies. This review also seeks to analyse results across a longer duration of follow-up, with subgroup and sensitivity analyses that were not carried out in previous studies.

\section{METHODS}

The review was reported in accordance with Preferred Reporting Items for Systematic Reviews and MetaAnalyses (PRISMA) guidelines. An expert team comprising medical librarians and biostatisticians from the Yong Loo Lin School of Medicine at National University of Singapore and clinicians from the Otorhinolaryngology Department at Tan Tock Seng Hospital was consulted to determine the overarching goals of our systematic scoping review, as well as the population and contexts to be evaluated.

Guided by the Population, Intervention, Comparison, Outcome and Study Design framework, the primary research question was determined as "How efficacious is HIFU as a treatment for benign thyroid nodules?" while the secondary research question was: "How safe is HIFU as a treatment for benign thyroid nodules?".

The study protocol was registered with the International Prospective Register of Systematic Reviews and available online ${ }^{11}$ (CRD42021254290). No amendments were made to the protocol subsequently. The inclusion and exclusion criteria for the review are outlined in Table 1.

\section{Literature search}

The PubMed, Embase and Cochrane databases were searched to retrieve articles that evaluated the efficacy and safety of HIFU as a treatment for benign thyroid nodules. The search used Boolean operators AND, OR and search terms used were "high intensity focused ultrasound", "focused ultrasound", "thyroid nodule", "thyroid neoplasm", "high intensity focused ultrasound OR focused ultrasound AND thyroid nodule OR thyroid neoplasm". Searches were confined to articles published between 1 January 1990 and 30 April 2021. All articles published in English or as English translations were included. The search was carried out between 28 April 2021 and 2 May 2021. The search process is summarised in the PRISMA flowchart (Supplementary Fig. S1 in the online version of this article).

Two reviewers (CWSC and AXYJ) of the research team independently reviewed the titles and abstracts identified by each database to identify the list of articles to be reviewed. If a consensus was not reached by the 2 reviewers, the article was reviewed by 4 other reviewers.

All reviewers then assessed each study using the Newcastle Ottawa Scale (NOS), ${ }^{12}$ acknowledged by the Cochrane Collaboration ${ }^{13}$ for evaluating the risk of bias at the outcome level. This was graded on a 9-star scale. Studies with $<5$ stars, 5-7 stars and $\geq 8$ stars were graded as having a high, moderate and low risk of bias, respectively. ${ }^{12}$

\section{Data extraction}

The following headings were used to extract data from all included articles (Table 2).

\section{Statistical analysis}

The main outcomes of this study were the VRRs of the treated nodule at 3, 6 and 24 months, pain scores pre- and post-procedure. The VRR was calculated based on the formula:

(baseline volume - volume at visit) (baseline volume) x 100 
Table 1. Population, intervention, comparison, outcome and study design (PICOS)

\begin{tabular}{|c|c|c|}
\hline PICOS & Inclusion criteria & Exclusion criteria \\
\hline Population & $\begin{array}{l}\text { Benign thyroid nodules or neoplasms } \\
\text { Age older than } 18 \\
\text { Confirmation of benign findings in ultrasound-guided } \\
\text { biopsies }\end{array}$ & $\begin{array}{l}\text { Extra-thyroidal tissue } \\
\text { Malignancy }\end{array}$ \\
\hline Intervention & HIFU & $\begin{array}{l}\text { Radiofrequency ablation } \\
\text { Other non-ultrasound therapies }\end{array}$ \\
\hline Comparison & $\begin{array}{l}\text { Comparisons of the number of nodules ablated by HIFU, } \\
\text { mean size (volume) of ablated nodules, device used, } \\
\text { amount of energy delivered per treatment, treatment time, } \\
\text { complications, VRR and pain scores at any time points } \\
\text { after treatment }\end{array}$ & \\
\hline Outcome & $\begin{array}{l}\text { Complete follow-up data on the number of nodules ablated } \\
\text { by HIFU, mean size (volume) of ablated nodules, device } \\
\text { used, amount of energy delivered per treatment, treatment } \\
\text { time, complications, VRR and pain scores at any time } \\
\text { points after treatment }\end{array}$ & \\
\hline \multirow[t]{5}{*}{ Study design } & $\begin{array}{l}\text { Articles in English or translated to English } \\
\text { Randomised controlled trials, cohort studies, case-control } \\
\text { studies, cross-sectional studies }\end{array}$ & $\begin{array}{l}\text { Grey literature, as electronic or print information not } \\
\text { published by commercial scholarly publishing } \\
\text { Case reports or series including fewer than } 10 \text { patients }\end{array}$ \\
\hline & $\begin{array}{l}\text { Years of publication: } 1 \text { January 1990-30 April } 2021 \\
\text { PubMed, Embase, Cochrane databases }\end{array}$ & $\begin{array}{l}\text { Reviews, meta-analyses, descriptive papers, letters, } \\
\text { editorials, conference abstracts, guidelines, consensus } \\
\text { statements }\end{array}$ \\
\hline & & $\begin{array}{l}\text { Studies with or likely with overlapping populations of } \\
\text { patients studied }\end{array}$ \\
\hline & & Studies focusing on non-human subjects \\
\hline & & $\begin{array}{l}\text { Studies with insufficient data on volume reduction of the } \\
\text { treated nodules }\end{array}$ \\
\hline
\end{tabular}

HIFU: high-intensity focused ultrasound; VRR: volume reduction rate

Meta-analytic pooling was performed using inverse variance for calculating weights, and Der SimonianLaird random effects modelling was utilised to determine the pooled proportions and $95 \%$ confidence intervals (CI). The $\mathrm{I}^{2}$ statistic for the pooled estimates was employed to determine heterogeneity among studies: slight (0-40\%), moderate (30-60\%), substantial $(50-90 \%)$ or considerable $(75-100 \%)$ heterogeneity, with $P<0.1$ indicating significant heterogeneity. ${ }^{14}$ The $P<0.1$ threshold was employed given the low statistical power of the chi-square test. We repeated the meta-analyses in subgroups in order to explore the sensitivity of our results to the same study characteristics. All statistical analyses were performed using RevMan 5.4 software (Cochrane, London, UK). ${ }^{13}$

\section{RESULTS}

The search from the 3 databases retrieved 139 articles. There were $99(71.2 \%)$ articles remaining after removal of 40 duplicates. After reviewing the titles and abstracts, $49(35.3 \%)$ articles were included. After final review, $9(6.5 \%)$ articles were included for the systematic review and $6(4.3 \%)$ were included for the meta-analysis. Of the excluded articles, 13 (32.5\%) were likely to have overlapping populations, $10(25.0 \%)$ studies were conference abstracts, 5 (12.5\%) were not relevant, $3(7.5 \%)$ included more than 1 therapy, 3 (7.5\%) were reviews, $2(5 \%)$ were not conducted on humans, $1(2.5 \%)$ was not in English, 1 (2.5\%) could not be retrieved, $1(2.5 \%)$ was a study record of an ongoing clinical trial and $1(2.5 \%)$ was a case report. An additional search of their bibliographies did not return any relevant studies.

\section{Characteristics of included studies}

Supplementary Table S1 (online version of this article) summarises the characteristics of the 9 included studies. Three were prospective studies and 6 were retrospective 
Table 2. Headings used to extract data from included studies

\begin{tabular}{|l|}
\hline Study characteristics \\
\hline Author \\
\hline Year of publication \\
\hline Institution at which the patients were treated \\
\hline Sample size \\
\hline Study design \\
\hline Demographic and clinical characteristics of included patients \\
\hline Mean age \\
\hline Sex \\
\hline Nodule characteristics \\
Size \\
Composition \\
\hline Pathology \\
\hline HIFU technique \\
\hline Mean treatment time \\
\hline Mean number of HIFU sessions \\
\hline Sedation and analgesia used \\
\hline Mean power delivered per nodule \\
\hline Type of HIFU device \\
\hline Outcomes \\
\hline Volume reduction rate \\
\hline Pain score pre- and post-procedures \\
\hline Major and minor complications \\
\hline Repeat HIFU procedure or need for subsequent surgery \\
\hline
\end{tabular}

HIFU: high-intensity focused ultrasound

studies. All were cohort studies. Six (66.7\%) studies were from Europe and $3(33.3 \%)$ studies were from Asia. The total energy of HIFU was in the range of $2.1-24.5 \mathrm{~kJ}$ and the duration of HIFU was in the range of $9.00-92.33 \mathrm{~min}$. The mean age of patients in the studies was in the range of 44.5-62.0 years. All nodules were confirmed as benign by fine needle aspiration cytology. The mean volume of the target nodules was $10.3 \mathrm{~mL}$ (range 1.3-39.2). Eight studies described the compositions of the treated nodules, with all being solid or predominantly solid with cystic portions $<30 \%$. Most patients underwent 1 session HIFU, with only 3 patients undergoing 2 sessions in 1 study. ${ }^{8}$ All of the included studies used the same device for HIFU but 3 studies used the latest Beamotion software (Theraclion SA, France)., ${ }^{4,15}, 16$ The mean follow-up period of these studies was 12.9 months. Based on the NOS scale, all studies had a moderate risk of bias (Supplementary Table S2).

\section{EFFICACY}

Follow-up schedules of these studies were not standardised. The pooled VRRs at 3, 6, and 24 months after HIFU were $42.14 \%$ (95\% CI 28.66-55.62, $\left.\mathrm{I}^{2}=91 \%\right) \quad$ (Fig. 1), $53.51 \%$ (95\% CI 36.78-70.25, $\mathrm{I}^{2}=97 \%$ ) (Fig. 2) and $46.89 \%$ (95\% CI 18.87-74.92, $\mathrm{I}^{2}=99 \%$ ), respectively (Fig. 3), indicating considerable heterogeneity. No studies recorded complete disappearance of the nodules. Two studies (22.2\%) divided nodules into 3 groups by their baseline volumes. ${ }^{16,17}$ Each group was analysed separately. Publication bias was not evaluated due to the small number of included studies.

Subgroup analyses (Supplementary Figs. 2-7) were stratified by categorical study-level characteristics including nodule size, treatment time and depthindependent acoustic energy. Subgroup and sensitivity analyses were performed for VRR at 3 months and 6 months. Only subgroup analysis was done for VRR at 24 months as only $3(33.3 \%)$ studies were included. From the analyses, it was found that the source of heterogeneity for 3-month VRR stratified by nodule size and treatment time was the study by Monpeyssen et al. ${ }^{4}$ Subsequent subgroup analyses after removal of Monpeyssen et al. ${ }^{4}$ were reported in Supplementary Figs. 8-13.

Lang et al. ${ }^{15,16}$ reported several statistically significant predictive factors for success including pre-ablation volume, total on-beam time and total mean energy delivered.

\section{Safety}

Pain, skin changes and oedema were the most common minor side effects of HIFU, which regressed spontaneously without any intervention. Six (66.7\%) out of the 9 studies included in this review reported pain as a common side effect. ${ }^{4,8,9,15,16,18}$ This was scored either based on a visual analogue scale ${ }^{4,8,9,15,16}$ or a numeric rating scale. ${ }^{18}$ The mean pain score during and post-procedure were 4.85 and 1.54 , respectively. The peri-procedure pain scores ranged from 1 to 7 (range 0-10). Patients experienced the most pain during the treatment, decreasing to post-procedure pain scores ranging from 0 to 4 (range $0-10$ ). One study recorded a change in symptoms from a baseline score of 4.12 (standard deviation [SD] 1.22) to 2.57 (SD 1.35) at 6 months. ${ }^{15}$ This was also recorded based on a visual analogue scale. One study reported that all patients experienced throat discomfort due to the probe pressure and cooling, and they described this as a spreading of pain towards the neck, scapula, trapezius muscle or arm over time. ${ }^{18}$ Another study reported that a patient 


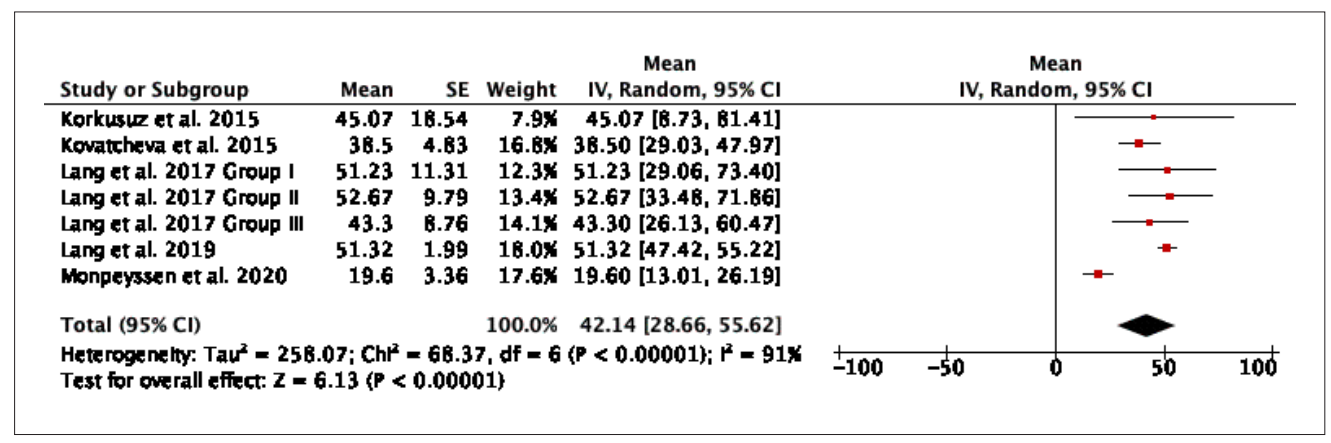

Fig. 1. Volume reduction rate at 3 months.

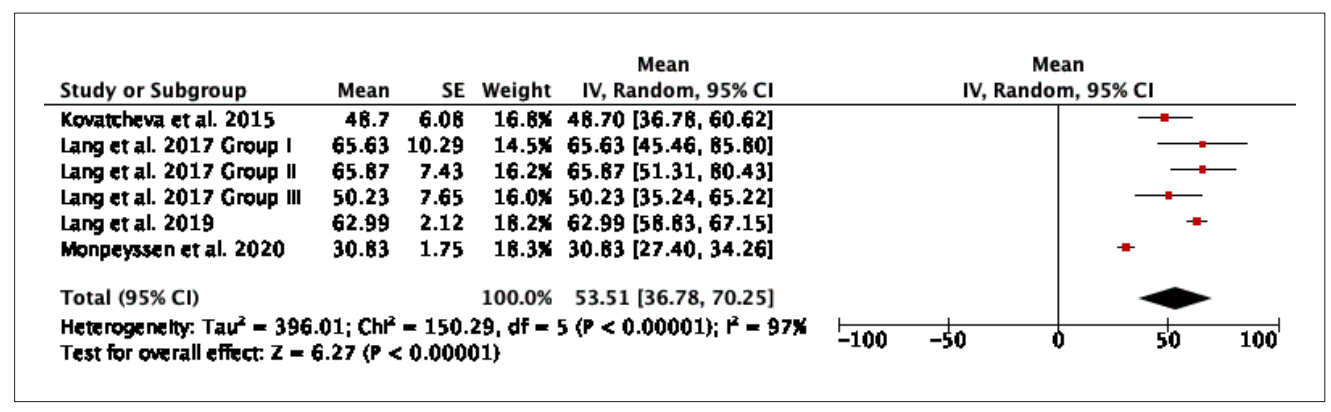

Fig. 2. Volume reduction rate at 6 months.

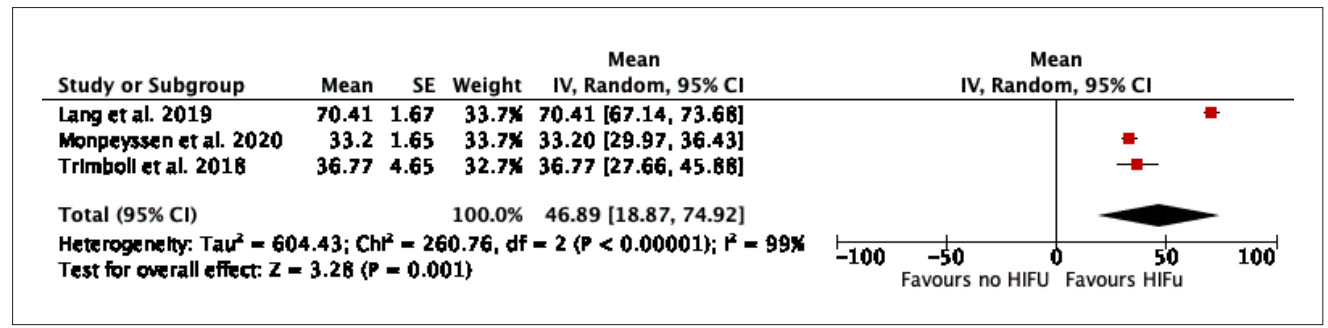

Fig. 3. Volume reduction rate at 24 months.

experienced severe shoulder pain that resolved within 6 months. $^{4}$

A major complication from HIFU treatment includes vocal cord paresis and voice hoarseness, which were reported in 2 studies. ${ }^{9}, 15$ Overall, 5/346 patients $(0.014 \%)$ experienced vocal cord palsy but all were transient. Lang et al. reported vocal cord paresis in $3(2.78 \%)$ patients that resolved within 3 months, ${ }^{15}$ whereas Prakash et al. reported voice hoarseness in $2(20 \%)$ patients that resolved after 10 days. ${ }^{9}$ Direct damage to the recurrent laryngeal nerve is thought to be caused by high temperatures $\left(60-80^{\circ} \mathrm{C}\right)$ during the HIFU procedure. ${ }^{18}$ However, indirect damage presenting as delayed vocal cord hoarseness may also occur due to local oedema of the surrounding structures. ${ }^{9}$ Hence, the amount of energy chosen and temperature reached is crucial to prevent both direct and indirect vocal cord palsy. Nonetheless, the main technical challenge of
HIFU is limiting damage to skin and subcutaneous fat while delivering sufficient heating for adequate nodule necrosis. ${ }^{18}$ In a recent study on small nodule ablation, temperature cut-off for cellular necrosis was achieved with very low energy $2.1 \mathrm{~kJ}$ (SD 1.1), resulting in a VRR of $48 \%$ at 12 months. ${ }^{19}$

Another major complication from HIFU is Horner's syndrome. In $2(22.2 \%)$ studies, $2 / 346$ patients $(0.5 \%)$ were reported to have Horner's syndrome but the ptosis improved gradually over a period of 6 months following treatment. ${ }^{4}, 15$ There were no other major side effects such as subcutaneous abscess, local blistering, thyroid dysfunction and nodal, tracheal or oesophageal rupture.

\section{DISCUSSION}

This systematic review and meta-analysis showed that pooled VRRs at 3, 6 and 24 months after HIFU were 42.14 (95\% CI 28.66-55.62, I ${ }^{2}=91 \%$ ) (Fig. 1), 53.51 
(95\% CI 36.78-70.25, $\mathrm{I}^{2}=97 \%$ ) (Fig. 2) and 46.89 (95\% CI 18.87-74.92, $\mathrm{I}^{2}=99 \%$ ) (Fig. 3), respectively.

It was noted that the VRR at 24 months is lower than that at 6 months. Only 2 studies $^{4,15}$ had a follow-up duration of more than 12 months. It remains unknown if the effects of HIFU on volume reduction remains in the long term, hence long-term studies are necessary.

Six out of the 9 studies ${ }^{4,9,15-17,20}$ referenced Lang et al. for the definition of treatment success as having VRR $\geq 50 \%$. However, based on our meta-analysis, only the 6-month VRR would have qualified as a success at $53.51 \%$, while the 3- and 24-month VRR at $42.41 \%$ and $46.89 \%$ would not qualify. In addition, given that multiple HIFU sessions were needed to attain successful ablation of the lesion, a contributing factor for the lack of success at 24 months could be the possibility of remnant lesional tissue that has continued to proliferate despite HIFU. Therefore, longer term results with more HIFU sessions are necessary to see if VRR is sustainable.

More important than treatment success is the correlation with patient satisfaction scores, which have not been addressed in most studies. Only 1 study reported patient satisfaction scores. ${ }^{7}$ In that study, the mean patient satisfaction score was 8.8 (SD 2.0, range 3-10) and was reported based on a visual analogue scale of $0-10$. This was an important determinant of the efficacy of HIFU treatment as it took into account all benefits and shortcomings of HIFU by including the patient perspective. With patient satisfaction scores, future HIFU efficacy studies may analyse how HIFU could cater to different patient populations.

Subgroup and sensitivity analyses showed that nodule size and treatment time were sources of heterogeneity for 3-month VRR. Another source of heterogeneity was the study by Monpeyssen et al. ${ }^{4}$ where a possible reason could be the significantly lower VRR at 3 months compared to other studies. This may be because the mean baseline volume was significantly higher than that in other studies. In large volume nodules, the peripheral areas are commonly excluded from treatment as they are close to vital structures, leading to a less complete ablation.

However, Monpeyssen et al. was the source of heterogeneity only for 3-month VRR stratified by nodule size and treatment time. This demonstrates that there are other factors for heterogeneity at play, which may largely be due to the insufficient number of studies on this topic, given that HIFU is a relatively new ablation technique for the treatment of benign thyroid nodules. This may also be due to the varied study designs included and the fact that all of the studies are cohort studies with different periods of intervention.

Another important consideration not addressed in our subgroup analysis is if the success rate is affected by nodule composition. Nodule composition is an important factor in determining the effectiveness of an ablation technique. It has been shown that HIFU is most effective at treating predominantly solid (cystic component $<30 \%$ ) benign thyroid nodules. ${ }^{5}$ In treating cystic nodules, the HIFU beam is partially reflected at the interface of the nodule. Thus the acoustical energy is not absorbed, reducing energy transfer and preventing the cyst-border wall from being treated. ${ }^{17}$ Moreover, small doses of energy are emitted at a time in HIFU and this may be distributed within a large volume of cyst liquid, limiting the temperatures that can be reached. Meanwhile, in RFA and microwave ablation (MWA), cysts within or infringing the effect radius may be sufficiently heated to ablate the adjacent cells. ${ }^{18}$

All the studies in this review included predominantly solid (cystic component $<30 \%$ ), or wholly solid and benign thyroid nodules. Hence subgroup analysis for nodule composition was not performed for this metaanalysis.

The revised American Thyroid Association guidelines recommend that surgical resection may be considered for benign solid or predominantly solid nodules that are either large ( $>4 \mathrm{~cm}$ in diameter) and/or cause compressive symptoms or clinical concern. ${ }^{2}$ In comparing HIFU with surgical reduction, the usual risks of surgery, including bleeding, infection and nerve palsies such as injuries to the recurrent laryngeal nerve appeared to be uncommon in HIFU based on our meta-analysis. Additionally, HIFU does not involve general anaesthesia and its associated risks, thus allowing for shorter hospital stays and lower costs per treatment (around USD350 per treatment). ${ }^{5}$

As compared to other minimally invasive ablation techniques, HIFU provides similar volume reduction rates. However, it is associated with longer treatment times and higher costs. On average, RFA or PEI would take $30-40 \%$ less time to completely ablate a wellselected $3 \mathrm{~cm}$ thyroid nodule while HIFU would have taken approximately $45-60 \mathrm{~min}$ for complete ablation. ${ }^{5}$ In addition, while the cost of disposables for HIFU is about half of 1 electrode of RFA or 1 antenna of MWA, investing in a HIFU device would cost around 12 times more than a RFA or MWA device. ${ }^{2}$ This would be a significant financial investment for any medical department and would imply greater treatment costs for patients. 
An important drawback of HIFU is its higher pain scores despite the utilisation of sedation and/or analgesia during the procedure, as was seen in 7 out of the 9 included studies. ${ }^{4,8,9,15,16,18,20}$ Based on our meta-analysis, the mean pain score during and post-procedure were 4.85 and 1.54 , respectively. The peri-procedure pain scores ranged from 1 to 7 (range $0-10$ ). This was higher than those reported in 2 other studies. ${ }^{21,22}$

\section{Limitations}

Firstly, our study may be limited by publication bias as results that suggest a benefit of HIFU were more likely to be published than those that do not. Secondly, the small number of studies and small sample sizes precluded our ability to adequately analyse subgroups and identify potentially important covariates. A further meta-analysis that considers the factors that cause heterogeneity may be needed when sufficient papers have been published in the future. Moreover, different studies had different follow-up durations. Thus, direct comparisons could not be drawn.

Thirdly, many studies reported medians and their ranges only. We converted those values to means and standard deviations using the formulae of Wan et al.,23 which may have affected the accuracy of the values. Fourthly, we were unable to analyse the absolute difference in nodule volumes before and after HIFU due to the heterogeneity of the baseline mean target nodule volume.

Finally, there were insufficient (less than 10) studies to assess funnel plot asymmetry based on Cochrane recommendations. ${ }^{13}$ We included articles in English or were translated to English, which may have resulted in an overestimation or underestimation of results, given the selection bias towards populations whose studies were conducted in English.

\section{CONCLUSION}

In conclusion, the present meta-analysis provides a summary of the literature on the efficacy and side effects of HIFU for benign thyroid nodules. The results suggested that HIFU may be an effective and safe alternative treatment modality. Larger clinical trials with longer follow-up are needed to evaluate the effectiveness of this treatment.

Further studies with larger populations to assess the risks and benefits of using different energy combinations of HIFU based on size, type and location of nodule are required..$^{20}$ These findings may provide deeper insights into efficacy and safety and guide the future management of benign thyroid nodules with HIFU as the treatment modality.

\section{REFERENCES}

1. Gharib H, Papini E, Garber JR, et al. American Association of Clinical Endocrinologists, American College Of Endocrinology, Associazione Medici Endocrinologi medical guidelines for clinical practice for the diagnosis and management of thyroid nodules - 2016 Update. Endocr Pract 2016;22:622-39.

2. Haugen BR, Alexander EK, Bible KC, et al. 2015 American Thyroid Association Management Guidelines for adult patients with thyroid nodules and differentiated thyroid cancer: The American Thyroid Association Guidelines task force on thyroid nodules and differentiated thyroid cancer. Thyroid 2016;26:1-133.

3. Spartalis E, Karagiannis SP, Plakopitis N, et al. Single-session high-intensity focused ultrasound (HIFU) ablation for benign thyroid nodules: a systematic review. Expert Rev Med Devices 2020;17:759-71.

4. Monpeyssen H, Ben Hamou A, Hegedüs L, et al. High-intensity focused ultrasound (HIFU) therapy for benign thyroid nodules: a 3 -year retrospective multicenter follow-up study. Int J Hyperthermia 2020;37:1301-9.

5. Lang $\mathrm{BH}, \mathrm{Wu} \mathrm{ALH}$. High intensity focused ultrasound (HIFU) ablation of benign thyroid nodules - a systematic review. J Ther Ultrasound 2017;5:11.

6. Esnault O, Franc B, Monteil JP, et al. High-intensity focused ultrasound for localized thyroid-tissue ablation: preliminary experimental animal study. Thyroid 2004;14:1072-6.

7. Kovatcheva RD, Zaletel K. High-intensity focused ultrasound for thyroid nodule ablation: The evidence to date. Reports in Medical Imaging 2017;10:9-16.

8. Kovatcheva RD, Vlahov JD, Stoinov JI, et al. Benign solid thyroid nodules: US-guided high-intensity focused ultrasound ablation Initial clinical outcomes. Radiology 2015;276:597-605.

9. Prakash PS, Oh HB, Tan WB, et al. The Efficacy and Safety of High-Intensity Focused Ultrasound (HIFU) Therapy for Benign Thyroid Nodules-A Single Center Experience from Singapore. World J Surg 2019;43:1957-63

10. Chung SR, Baek JH, Suh CH, et al. Efficacy and safety of high-intensity focused ultrasound (HIFU) for treating benign thyroid nodules: a systematic review and meta-analysis. Acta Radiol 2020;61:1636-43.

11. Clarissa C, Au J, Gan J, et al. The efficacy and safety of high-intensity focused ultrasound (HIFU) in the treatment of benign thyroid nodules: A systematic review and meta-analysis from 1990 to 2021. PROSPERO 2021 CRD42021254290. Available at: https://www.crd.york.ac.uk/prospero/display record. php?ID=CRD42021254290. Accessed on 15 February 2022.

12. Wells GA, Shea B, O'Connell D, et al. The Newcastle-Ottawa Scale (NOS) for assessing the quality of nonrandomised studies in meta-analyses. Available at: http://www.ohri.ca/programs/clinical_ epidemiology/oxford.asp. Accessed on 4 February 2022.

13. Boutron I, Page MJ, Higgins JPT, et al. Chapter 7: Considering bias and conflicts of interest among the included studies. In: Higgins JPT, Thomas J, Chandler J et al. Cochrane Handbook for Systematic Reviews of Interventions version 6.2 (updated February 2021). Available at: https://training.cochrane.org/handbook/current/chapter07\#section-7-5. Accessed on 12 February 2022.

14. Deeks JJ, Higgins JPT, Altman DG. Chapter 9: Analysing data and undertaking meta-analyses. In: Higgins JPT, Green S. Cochrane Handbook for Systematic Reviews of Interventions Version 5.1.0. Available at: https://handbook-5-1.cochrane.org/chapter_9/9_5_2_ 
identifying_and_measuring_heterogeneity.htm. Accessed on 12 June 2021.

15. Lang BHH, Woo YC, Chiu KWH. Two-year efficacy of singlesession high-intensity focused ultrasound (HIFU) ablation of benign thyroid nodules. Eur Radiol 2019;29:93-101.

16. Lang BH, Woo YC, Chiu KW. Single-Session High-Intensity Focused Ultrasound Treatment in Large-Sized Benign Thyroid Nodules. Thyroid 2017;27:714-21.

17. Sennert M, Happel C, Korkusuz Y, et al. Further Investigation on High-intensity Focused Ultrasound (HIFU) Treatment for Thyroid Nodules: Effectiveness Related to Baseline Volumes. Acad Radiol 2018;25:88-94.

18. Korkusuz H, Fehre N, Sennert M, et al. Volume reduction of benign thyroid nodules 3 months after a single treatment with high-intensity focused ultrasound (HIFU). J Ther Ultrasound 2015;3:4.
19. Trimboli P, Bini F, Marinozzi F, et al. High-intensity focused ultrasound (HIFU) therapy for benign thyroid nodules without anesthesia or sedation. Endocrine 2018;61:210-5.

20. Trimboli P, Pelloni F, Bini F, et al. High-intensity focused ultrasound (HIFU) for benign thyroid nodules: 2-year follow-up results. Endocrine 2019;65:312-17.

21. Ugurlu MU, Uprak K, Akpinar IN, et al. Radiofrequency ablation of benign symptomatic thyroid nodules: prospective safety and efficacy study. World J Surg 2015;39:961-8.

22. Yan J, Qiu T, Lu J, et al. Microwave ablation induces a lower systemic stress response in patients than open surgery for treatment of benign thyroid nodules. Int J Hyperthermia 2018;34:606-10.

23. Wan X, Wang W, Liu J, et al. Estimating the sample mean and standard deviation from the sample size, median, range and/or interquartile range. BMC Med Res Methodol 2014;14:135. 